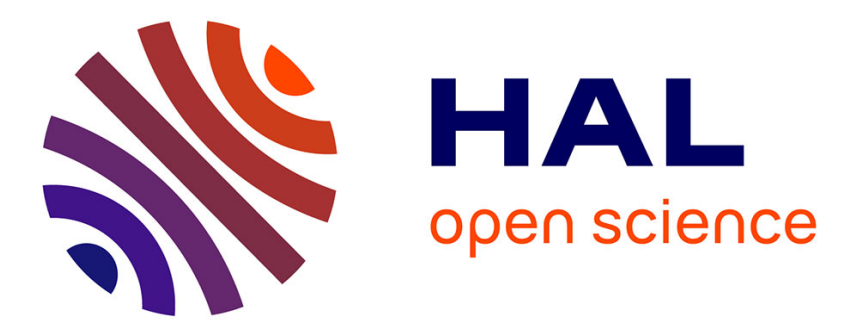

\title{
Influence de l'environnement sur la propagation des fissures courtes et longues dans un alliage léger type 7075
}

\author{
A. Zeghloul, J. Petit
}

\section{To cite this version:}

A. Zeghloul, J. Petit. Influence de l'environnement sur la propagation des fissures courtes et longues dans un alliage léger type 7075. Revue de Physique Appliquée, 1989, 24 (9), pp.893-904. 10.1051/rphysap:01989002409089300 . jpa-00246112

\section{HAL Id: jpa-00246112 https://hal.science/jpa-00246112}

Submitted on 1 Jan 1989

HAL is a multi-disciplinary open access archive for the deposit and dissemination of scientific research documents, whether they are published or not. The documents may come from teaching and research institutions in France or abroad, or from public or private research centers.
L'archive ouverte pluridisciplinaire HAL, est destinée au dépôt et à la diffusion de documents scientifiques de niveau recherche, publiés ou non, émanant des établissements d'enseignement et de recherche français ou étrangers, des laboratoires publics ou privés. 
Classification

Physics Abstracts

$46.30 \mathrm{~N}-62.20 \mathrm{M}$

\title{
Influence de l'environnement sur la propagation des fissures courtes et longues dans un alliage léger type 7075
}

\author{
A. Zeghloul (*) et J. Petit \\ Laboratoire de Mécanique et Physique des Matériaux, URA CNRS 863, E.N.S.M.A., 86034 Poitiers Cedex, \\ France
}

(Reçu le 27 juin 1988, révisé le 2 février 1989, accepté le 9 juin 1989)

\begin{abstract}
Résumé. - La propagation en fatigue des fissures courtes bidimensionnelles a été étudiée à l'air et dans le vide dans l'alliage 7075 T651 (vieilli au pic de durcissement) et à l'air pour deux rapports de charge dans l'alliage 7075 T7351 (sur-revenu). Les essais ont été effectués dans le domaine des basses vitesses de fissuration au voisinage du seuil de non-propagation. Sur la base des mesures de fermeture de fissure et des résultats relatifs à la propagation des fissures longues, il est montré que, pour un environnement donné, la propagation des fissures de petites dimensions peut être décrite à l'aide d'une seule relation liant la vitesse de fissuration au facteur d'intensité de contrainte effectif $\Delta K_{\text {eff }}$.
\end{abstract}

\begin{abstract}
The growth in fatigue of small bidimensionnal cracks has been studied in air and in vacuum on the 7075 T651 Aluminum alloy (peak aged) and in air at two load ratio, on the 7075 T7351 alloy (overaged). The low growth rate range was investigated near threshold conditions. On the basis of crack closure measurements and long cracks propagation results, it was shown that, for given environmental conditions, the behaviour of small and long cracks can be described in terms of the effective stress intensity factor range.
\end{abstract}

\section{Introduction.}

L'étude de l'influence de l'environnement sur la propagation des fissures courtes en fatigue a fait l'objet de peu de travaux en dehors de ceux consacrés aux environnements corrosifs $[1,2]$. Récemment Gerdes et al. [3] ont montré dans un alliage de titane type Ti-8,6 Al l'existence d'une propagation de microfissures de surface nettement plus rapide à l'air ambiant que sous vide. Par ailleurs Lankford [4] a suggéré l'absence d'effet d'environnement sur la propagation du même type de fissures dans le cas d'un alliage léger type 7075 T651 en comparant des résultats obtenus à l'air et sous azote purifié $\left(\sim 2\right.$ ppm $\left.\mathrm{H}_{2} \mathrm{O}\right)$. Toutefois Zeghloul et Petit [5] ont ensuite montré que la propagation des fissures courtes bidimensionnelles dans un alliage 7075 T7351 était observée à des niveaux de contraintes

(*) Depuis le 1-09-88: Laboratoire de Physique et Mécanique des Matériaux, URA CNRS 1215 ISGMP, 57045 Metz Cedex 1, France. bien plus faibles sous azote purifié $(\sim 3 \mathrm{ppm})$ que sous vide $\left(<5 \times 10^{-4} \mathrm{~Pa}\right)$, ces niveaux étant nettement inférieurs à ceux correspondant aux seuils de propagation des fissures longues dans les deux environnements.

La comparaison de ces résultats [4 et 5] à ceux obtenus précédemment pour des fissures longues sur le même alliage 7075 dans différentes conditions de revenu [6-8], suggère l'existence d'un effet fragilisant considérable induit par la vapeur d'eau de l'atmosphère environnante, cet effet pouvant être observé en présence de simples traces d'eau lorsque la propagation s'effectue à des vitesses suffisamment lentes $\left(<10^{-8} \mathrm{~m} /\right.$ cycle $)$.

Par ailleurs, différents auteurs [9-14] ont montré que l'effet de fissure courte (propagation plus rapide et seuil de propagation plus faible que pour les fissures longues) observé dans le cas de fissures bidimensionnelles obtenues par usinage de fissures longues, peut être décrit par la diminution de l'effet de fermeture lorsque la longueur du défaut diminue, sans que l'influence de l'environnement soit toutefois prise en compte [5]. 
Pour atteindre une meilleure compréhension de l'influence de l'environnement et du rôle de la microstructure sur la propagation des fissures de petites dimensions, une étude a été développée dans le prolongement des travaux antérieurs sur les fissures longues dans l'alliage 7075. Cet article présente et discute les résultats obtenus dans le cas de fissures bidimensionnelles se propageant à l'air et sous vide dans cet alliage dans les états de revenu T7351 et T651.

\section{Conditions et méthodes expérimentales}

La composition en pourcentage pondéral de l'alliage étudié est la suivante :

\section{Al-5,7 Zn-2,43 Mg-1,5 Cu-0,2 Cr-0,21 Fe $-0,16 \mathrm{Si}-0,04 \mathrm{Mn}$ et $\mathrm{Ti}$.}

Les propriétés mécaniques pour l'état T651 (vieilli au pic de durcissement) et l'état T7351 (sur-revenu) sont respectivement 527 et $444 \mathrm{MPa}$ pour la limite d'élasticité, 590 et $513 \mathrm{MPa}$ pour la limite de rupture, 11 et $11,7 \%$ pour l'allongement réparti. La taille moyenne des grains est de $40 \times 150 \times 600 \mu \mathrm{m}$ et les caractéristiques de la précipitation durcissante existant dans les deux états sont les suivantes : précipités fins dont la taille n'excède pas $6 \mathrm{~nm}$ et tous cohérents avec la matrice pour l'état T651 et précipités plus gros de taille 10 à $20 \mathrm{~nm}$ et présentant un écart à la cohérence pour l'état T7351.

Les essais de fissuration ont été effectués sur deux géométries d'éprouvettes :

- type CT avec une largeur $W=75 \mathrm{~mm}$ et une épaisseur $e=10 \mathrm{~mm}$

- type SEN de traction avec $W=18 \mathrm{~mm}$ et $e=6 \mathrm{~mm}$.

Ces éprouvettes ont été testées à la fréquence de $35 \mathrm{~Hz}$ sur une machine électrohydraulique équipée d'une chambre hermétique permettant les essais sous vide $\left(<5 \times 10^{-4} \mathrm{~Pa}\right)$. Après préfissuration jusqu'au seuil de non-propagation, les éprouvettes ont été usinées selon la procédure indiquée dans les figures 1 (par fraisage) et 2 (par électroérosion) pour obtenir un défaut traversant de 0,1 à $0,4 \mathrm{~mm}$ de longueur avec un front sensiblement linéaire (fissure courte bidimensionnelle). Le diamètre du fil d'usinage par électroérosion est de $0,2 \mathrm{~mm}$ conduisant à un rayon à fond d'entaille d'environ $0,15 \mathrm{~mm}$.

La longueur de fissure a été mesurée, sur chacune des faces de l'éprouvette, à l'aide d'un microscope de grossissement $G=200$ à 500 . Les mesures de fermeture de fissure ont été effectuées selon la méthode de variation de complaisance, les diagrammes effort-déplacement au cours d'un cycle étant enregistrés à l'aide d'un capteur de déplacement à lames, placé de part et d'autre de l'entaille. Dans le cas de l'éprouvette CT, la sensibilité de la mesure de

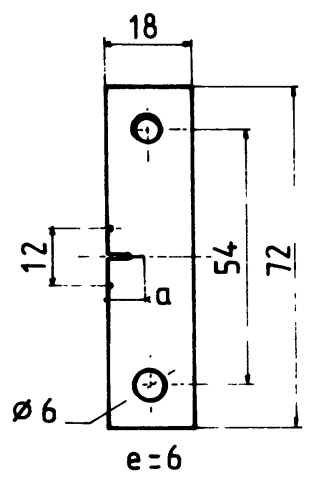

a)

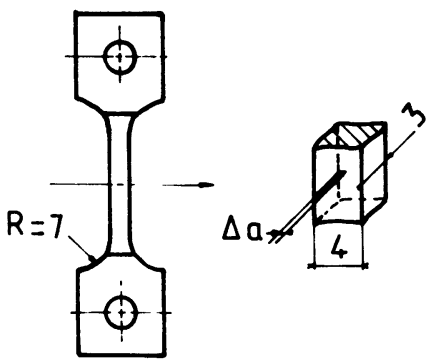

b)
Fig. 1. - Eprouvette SEN utilisée pour l'obtention d'une fissure courte traversante : a) éprouvette SEN pour préfissuration ; b) éprouvette usinée.

[SEN specimen used to obtain through thickness short cracks : a) SEN specimen for precracking ; b) machined specimen.]

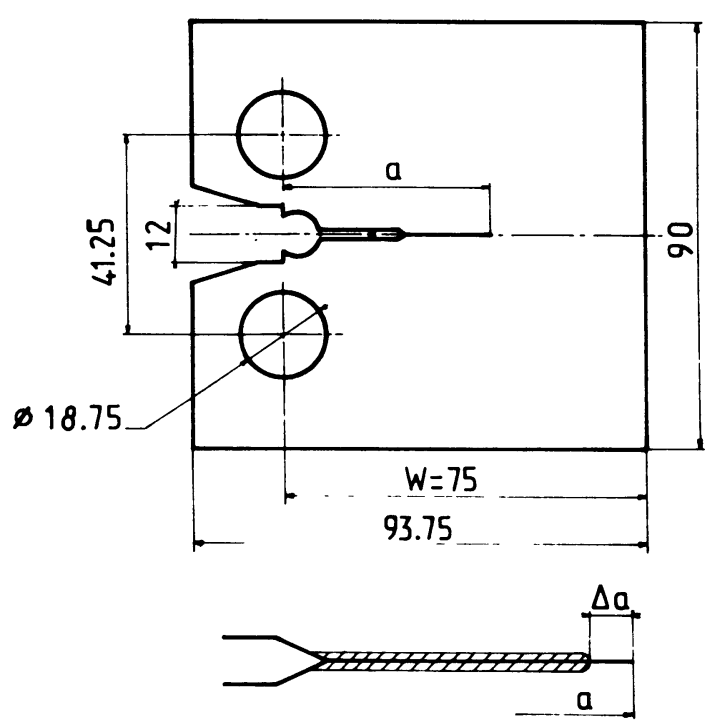

Fig. 2. - a) Eprouvette CT pour préfissuration; b) Eprouvette usinée par électroérosion pour obtenir une fissure courte.

[a) CT specimen for precracking; b) machined specimen (sparkling) to obtain a short through crack.]

fermeture a été optimisée en effectuant la préfissuration sur une longueur $a$ telle que $a / W=0,6$.

La valeur du facteur d'intensité de contrainte $K$ a été calculée en assimilant l'entaille à la fissure, à partir de la relation polynomiale suivante [15] :

$$
K=\frac{P}{e \sqrt{W}} f(a / W)
$$

avec

$P \quad=$ charge appliqué ;

$e, W \quad=$ épaisseur et largeur d'éprouvette; 


$$
\begin{aligned}
f(a / W)= & 29,6(a / W)^{1 / 2}-185,5 \\
& (a / W)^{3 / 2}+655,7(a / W)^{5 / 2}-1017 \\
& (a / W)^{7 / 2}+638,9(a / W)^{9 / 2} \text { pour les } \\
& \text { éprouvettes CT } ; \\
\text { et } f(a / W)= & 1,99(a / W)^{1 / 2}-0,41(a / W)^{3 / 2}+18,7 \\
& (a / W)^{5 / 2}-38,48(a / W)^{7 / 2}+53,85 \\
& (a / W)^{9 / 2} \text { pour les éprouvettes SEN } \\
& \text { à extrémités libres en rotation. }
\end{aligned}
$$

Un calcul aux éléments finis utilisant au voisinage de la pointe de fissure des éléments de transition à 8 nœuds [16] a permis de vérifier la validité de ces relations pour nos éprouvettes. Le facteur $K$ a été déterminé à partir de l'estimation de l'intégrale $J$ [17] moyennée sur trois contours.

\section{Essais de référence pour les fissures longues.}

Les essais de référence pour les fissures longues proviennent essentiellement des travaux antérieurs effectués sur les mêmes alliages [6,7, 18-21]. Les principaux résultats, présentés ci-dessous, serviront de base à l'analyse du comportement des fissures courtes.

3.1 INFLUENCE DE LA MICROSTRUCTURE. - La caractérisation de l'influence spécifique de la microstructure sur la fissuration en fatigue à basse vitesse a été étudiée sous vide $[7,18,22]$ en considérant un alliage 7075 dans différents états de revenu. Les résultats obtenus dans les états T651 et T7351 sont présentés dans la figure 3 . Les essais ont été conduits sur des éprouvettes CT $(W=75 \mathrm{~mm} ; e=10 \mathrm{~mm})$ à $35 \mathrm{~Hz}$ et $R=0,1$ sous vide $<5 \times 10^{-4} \mathrm{~Pa}$.

Dans le domaine des vitesses moyennes ( $>10^{-8} \mathrm{~m} /$ cycle), les deux alliages présentent un comportement assez similaire. Par contre, au voisinage du seuil de propagation, l'alliage T651 présente une résistance à la fissuration nettement supérieure à celle de l'alliage sur-revenu T7351. L'observation microfractographique des faciès de rupture montre que le mécanisme de fissuration, identique à vitesse moyenne, devient très différent près du seuil (Fig. 4).

Les travaux antérieurs [22-24] ont montré que la propagation de type transgranulaire dans les deux alliages, peut être analysée comme suit :

- Dans l'état sur-revenu T7351, on observe une déformation plastique en pointe de fissure de type homogène faisant intervenir différents systèmes de glissement simultanément ; ce mécanisme est favorisé par la présence de précipités de taille assez importante $(\sim 20 \mathrm{~nm})$ qui résistent au cisaillement. En outre l'appauvrissement relatif de la matrice en $\mathrm{Cu}$ et $\mathrm{Mg}$ peut favoriser le glissement dévié en jouant sur l'énergie de défaut d'empilement [24]. Il en résulte un endommagement assez rapide, localisé autour des précipités. Les faciès de rupture en

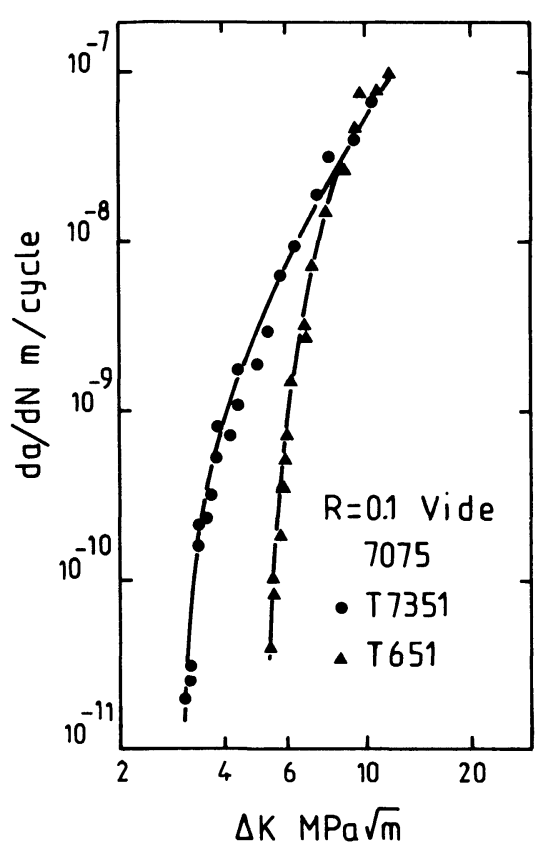

Fig. 3. - Vitesses de propagation en fatigue $\mathrm{d} a / \mathrm{d} N$ en fonction de $\Delta K$ pour des fissures longues dans les alliages d'aluminium 7075 T651 et T7351 sollicités sous vide (éprouvette CT).

[Fatigue crack growth rates $\mathrm{d} a / \mathrm{d} N$ versus $\Delta K$ for long cracks in 7075 T651 and T7351 aluminium alloys in vacuum (CT specimens).]

fatigue associés à ce type de propagation qui est contrôlée par les dispersoïdes sont macroscopiquement et microscopiquement assez plats (Fig. 4a et c) essentiellement marqués par le sillage des gros précipités intermétalliques qui, à basse vitesse, sont également contournés avant rupture (Fig. 4a).

- Dans l'état T651, un mécanisme analogue est observé à vitesse moyenne lorsque la contrainte est suffisamment élevée pour que la déformation reste homogène et concerne une zone portant sur plusieurs grains à la fois. La propagation est alors contrôlée par la précipitation $M^{\prime}\left(\mathrm{Mg}_{4} \mathrm{Zn}_{11} \mathrm{Al}\right.$ en plaquettes $1,5 \times 5,0 \mathrm{~nm}) \quad[18]$. A vitesse lente $\left(<10^{-9} \mathrm{~m} /\right.$ cycle $)$, après un domaine de transition où les mesures sont fortement dispersées [20] la déformation devient hétérogène et localisée en un seul grain en pointe de fissure. Un mécanisme de glissement planaire (également observé dans les zones GP de l'alliage sous revenu [7]) prédomine, favorisé par la présence de précipités fins cisaillables (zones GP) et des précipités $\mathbf{M}^{\prime}$ dont la cohérence autorise le cisaillement. En outre les solutions solides riches en $\mathrm{Cu}$ et $\mathrm{Mg}$ réduisent le glissement dévié [24]. Les faciès de rupture associés à ce mécanisme sont caractérisés par leur aspect cristallographique, présentant de larges facettes fortement désorientées qui génèrent des surfaces à forte rugosité $[7,20]$ (Fig. $4 b$ et d). 


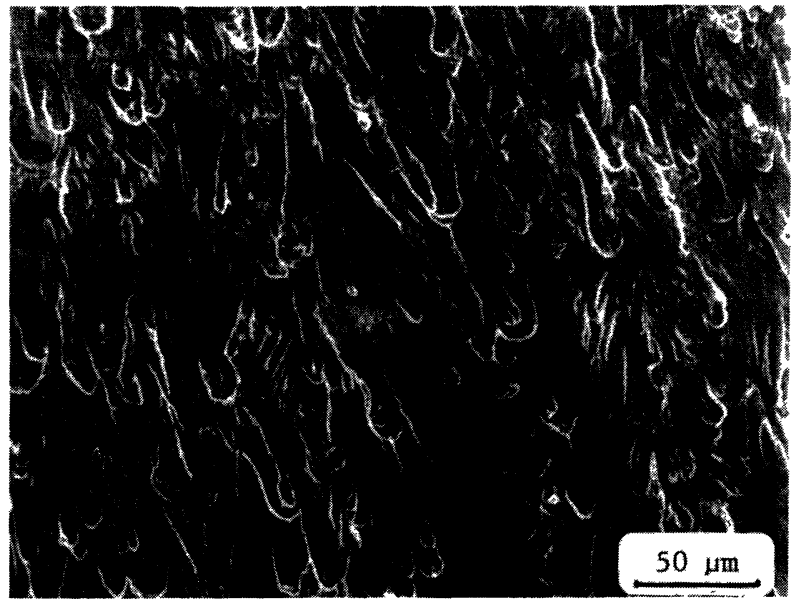

a)

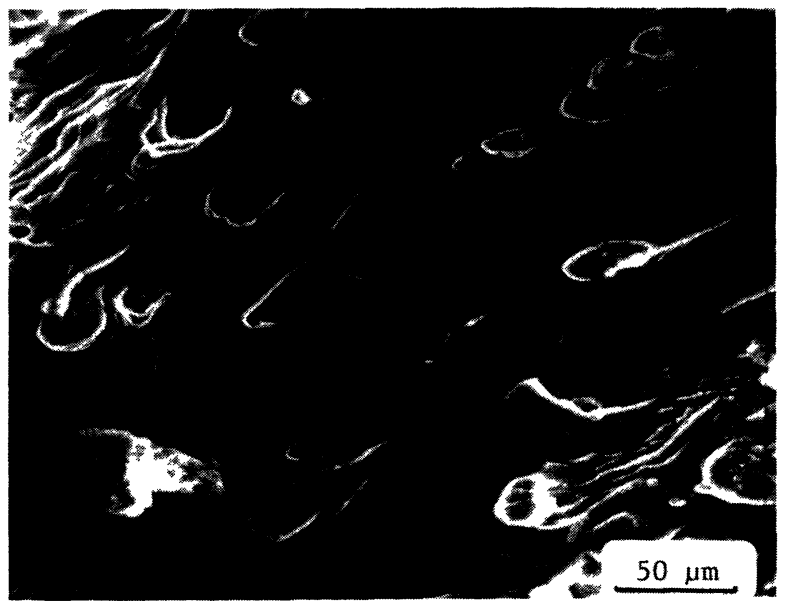

b)

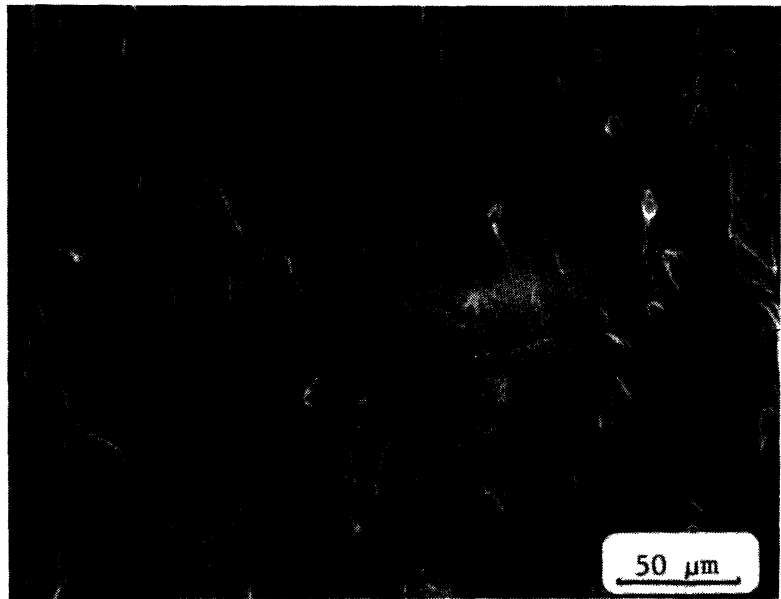

c)

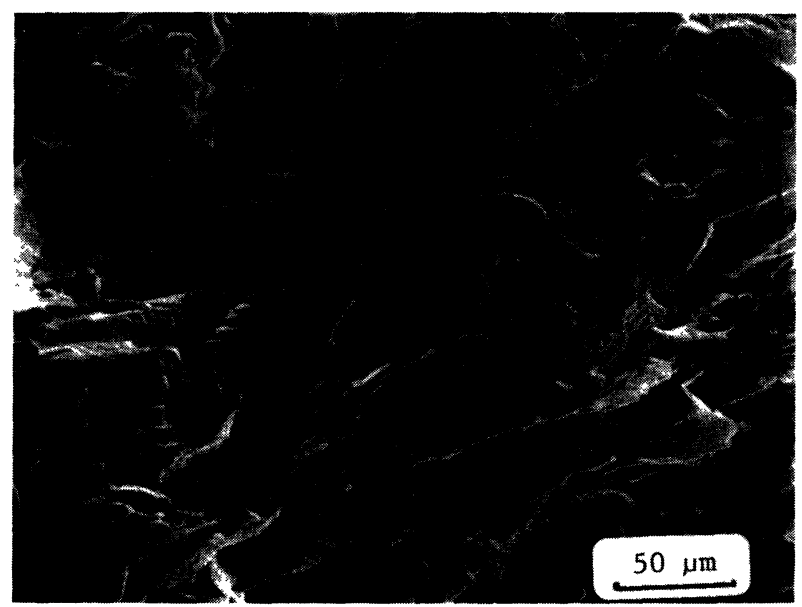

d)

Fig. 4. - Faciès micrographiques de la surface de rupture de l'alliage 7075 sollicité sous vide: a) T7351 $\mathrm{d} a / \mathrm{d} N=3 \times 10^{-8} \mathrm{~m} /$ cycle ; b) $\mathrm{T} 651 \mathrm{~d} a / \mathrm{d} N=2 \times 10^{-8} \mathrm{~m} /$ cycle ; c) T7351 au seuil de non-propagation; d) T651 au seuil de non-propagation.

[Microfractographic aspect of the cracked surface of 7075 specimens tested in vacuum: a) $\mathrm{T} 735, \mathrm{~d} a / \mathrm{d} N=$ $3 \times 10^{-8} \mathrm{~m} /$ cycle ; b) T651 $\mathrm{d} a / \mathrm{d} N=2 \times 10^{-8} \mathrm{~m} /$ cycle ; c) $\mathrm{T} 7351$, at threshold ; d) $\mathrm{T} 651$, at threshold.]

Les mesures de fermeture effectuées par variation de complaisance ont permis d'établir les diagrammes $\mathrm{d} a / \mathrm{d} N-\Delta K_{\text {eff }}$ (Fig. 5). Ces résultats, couplés à ceux obtenus sur d'autres alliages légers, ont permis de décrire le comportement sous vide des alliages d'aluminium à l'aide du modèle proposé par Weertman [25] qui repose sur le concept de cumul de dommage par déformation plastique cyclique en pointe de fissure. Une loi du type suivant a été proposée :

$$
\frac{\mathrm{d} a}{\mathrm{~d} N}=\frac{A \Delta K_{\mathrm{eff}}^{4}}{\mu \sigma^{2} U}
$$

avec

$A=$ constante sans dimension;

$\mu=$ module de cisaillement ;

$\sigma=$ contrainte d'écoulement plastique ;

$U=$ énergie de création d'une nouvelle unité de surface de fissure.
L'influence de la microstructure peut être prise en compte de deux façons différentes : soit un changement de valeur de l'énergie de création de surface $U$, un mécanisme cristallographique étant alors associé à une énergie nettement plus grande ; soit un effet micromécanique lié à la déviation localisée de la pointe de fissure selon des modèles tels que celui proposé par Suresh [26] et qui introduisent un coefficient correcteur $\chi(\chi<1)$ qui réduit le facteur d'intensité de contrainte réel $\left(\Delta K_{\text {eff }}=\chi \Delta K_{\text {eff }}\right)$.

Les deux approches ne sont pas incompatibles. Après application de la correction de Suresh (avec $\chi \sim 0,73$ pour des déviations de $45^{\circ}$ ), on obtient une courbe $\mathrm{d} a / \mathrm{d} N-\Delta K_{\text {eff }}$ unique (Fig. 5) qui conduit à une valeur du seuil effectif sous vide, c'est-à-dire du seuil intrinsèque $\Delta K_{i}$, de l'ordre de $2 \mathrm{MPa} \sqrt{m}$ [21]. Il est important de noter que cette valeur seuil semble indépendante de l'état microstructural et, 


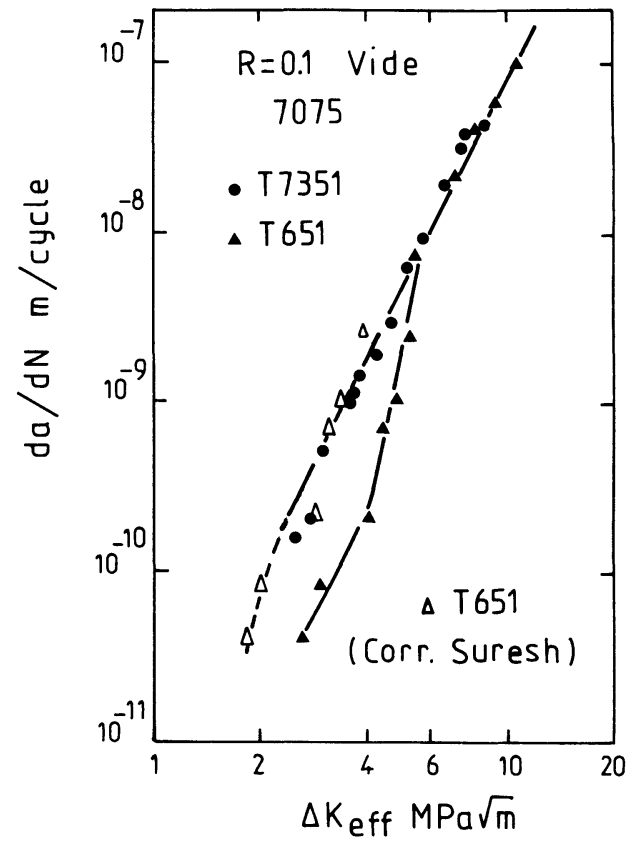

Fig. 5. $-\mathrm{d} a / \mathrm{d} N-\Delta K_{\text {eff }}$ sous vide pour l'alliage 7075 dans les états T7351 et T651 après correction de Suresh.

[d $a / \mathrm{d} N$ vs. $\Delta K_{\text {eff }}$ in vacuum for $7075 \mathrm{~T} 7351$ and T651 after Suresh's correction.]

donc, serait ainsi essentiellement une caractéristique du métal de base.

\subsection{INFLUENCE DE L'ENVIRONNEMENT AMBIANT.}

- Les figures $6 \mathrm{a}$ et $6 \mathrm{~b}$ comparent la propagation dans l'air ambiant à celle observée sous vide pour les deux nuances de l'alliage 7075 . Les essais ont été conduits dans les mêmes conditions que sous vide (même rapport de charge $R=0,1$, même type d'éprouvette...).

A vitesse moyenne l'influence de l'environnement est faible. Par contre, dans l'intervalle de vitesse compris entre $10^{-9}$ et $10^{-8} \mathrm{~m} /$ cycle, on observe l'apparition à l'air d'un effet d'environnement qui réduit la résistance à la fissuration de l'alliage. Cet effet est plus marqué dans l'état T651. Le comportement à l'air des deux nuances s'évère assez proche, le seuil dans l'état T651 étant cependant un peu plus élevé. L'examen des faciès montre l'existence d'un mécanisme similaire dans les deux cas et la présence d'important dépôts d'oxyde formés par «fretting » $[21,27,28]$. L'analyse SIMS [23] des surfaces a montré que la surépaisseur était plus importante dans le cas de l'alliage sur-revenu, cette surépaisseur devenant du même ordre que le $\Delta$ CTOD calculé [29] $(\mathrm{CTOD}=$ Crack tip Opening Displacement, déplacement d'ouverture à fond de fissure). Dans ce cas, on peut donc considérer que le niveau seuil est contrôlé par l'épaississement de l'oxyde, ce qui explique l'observation d'un seuil relativement élevé (identique au vide).

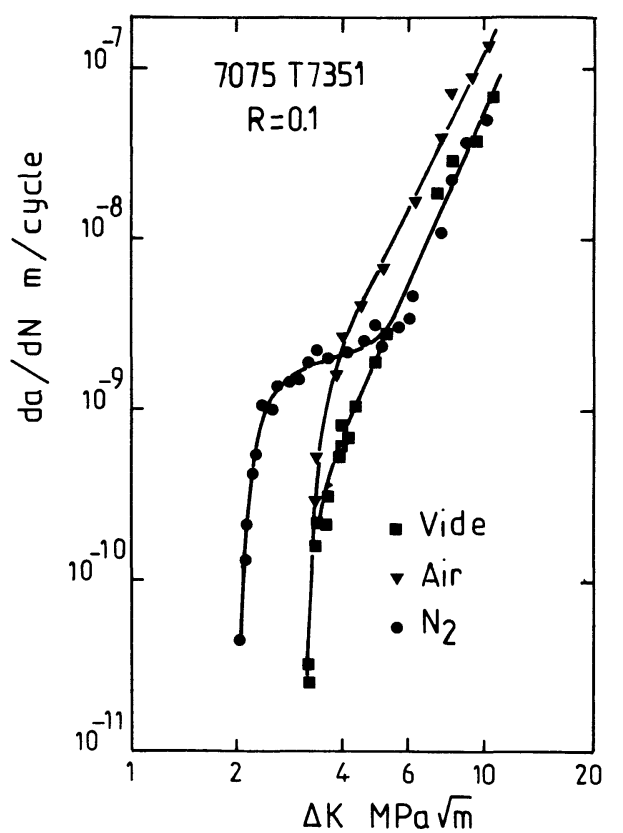

a)

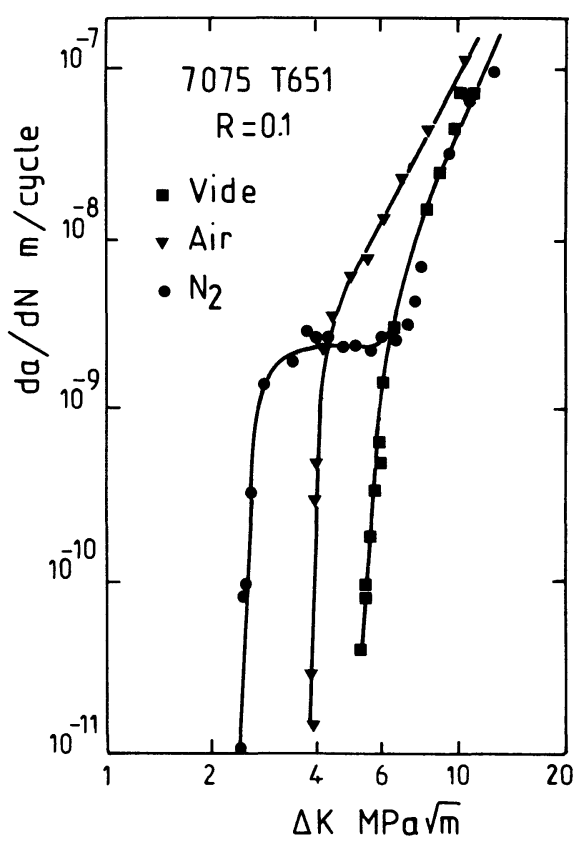

b)

Fig. 6. $-\mathrm{d} a / \mathrm{d} N-\Delta K$ à l'air, sous vide et dans une atmosphère d'azote contenant $30 \mathrm{ppm}$ d' $\mathrm{H}_{2} \mathrm{O}$ : a) 7075 T7351; b) 7075 T651.

[d $a / \mathrm{D} N$ vs. $\Delta K$ in air, vacuum and nitrogen containing 30 ppm $\mathrm{H}_{2} \mathrm{O}$; a) $7075 \mathrm{~T} 7351$; b) 7075 T651.]

Dans l'état T651, le seuil à l'air est inférieur à celui sous vide ce qui est cohérent avec un faible effet de coin et une influence marquée de l'environnement. Pour s'affranchir des effets d'oxydation, des essais ont été effectués sous azote contenant des traces d'eau (Fig. 6a et 6b). Des mesures récentes [30] ont montré que la teneur en eau du gaz dans l'environnement immédiat de l'éprouvette à l'intérieur de l'enceinte était de l'ordre de 15 ppm (la source étant 
un gaz purifié $<3 \mathrm{ppm}_{2} \mathrm{O}$ et $1 \mathrm{ppm} \mathrm{O}_{2}$ ). Les résultats se caractérisent par un comportement à vitesse moyenne analogue à celui sous vide et un comportement près du seuil qui correspond à une résistance inférieure à celle à l'air. Ce même résultat avait été initialement observé sur un alliage 2618 T651 [31, 32]. L'effet d'environnement qui se manifeste vers $10^{-9} \mathrm{~m} /$ cycle s'avère donc encore plus marqué qu'à l'air en présence de simples traces d'eau et d'oxygène.

Le diagramme $\mathrm{d} a / \mathrm{d} N-\Delta K_{\text {eff }}$ de la figure 7 est issu des mesures de fermeture effectuées au cours des essais en référence. On constate l'existence sous environnement actif, en particulier sous azote, d'un seuil effectif très bas, de l'ordre de $0,4 \mathrm{MPa} \sqrt{m}$ avec une forte dispersion des résultats à faibles vitesses. A l'air à $R=0,1$, des valeurs plus élevées du seuil effectif $\left(\Delta K_{\text {eff.s }}\right)$ sont obtenues, ce qui suggère une certaine inhibition de l'activité de l'environnement par les dépôts d'oxyde, lorsque le $\triangle$ CTOD devient très faible près du seuil. Sur la base des observations faites sur différents alliages [7] la diminution de la résistance à la fissuration en atmosphère humide a été analysée comme suit :

- à vitesses moyennes $\left(>10^{-8} \mathrm{~m} /\right.$ cycle $)$ le mécanisme de propagation est identique à celui observé sous vide, l'énergie $U$ étant simplement réduite par l'adsorption de vapeur d'eau (Effet Rhebinder) [33]. A l'air, l'effet est donc plus marqué que sous azote contenant des traces d'eau, ce dernier environnement ne se différenciant pas du vide ;

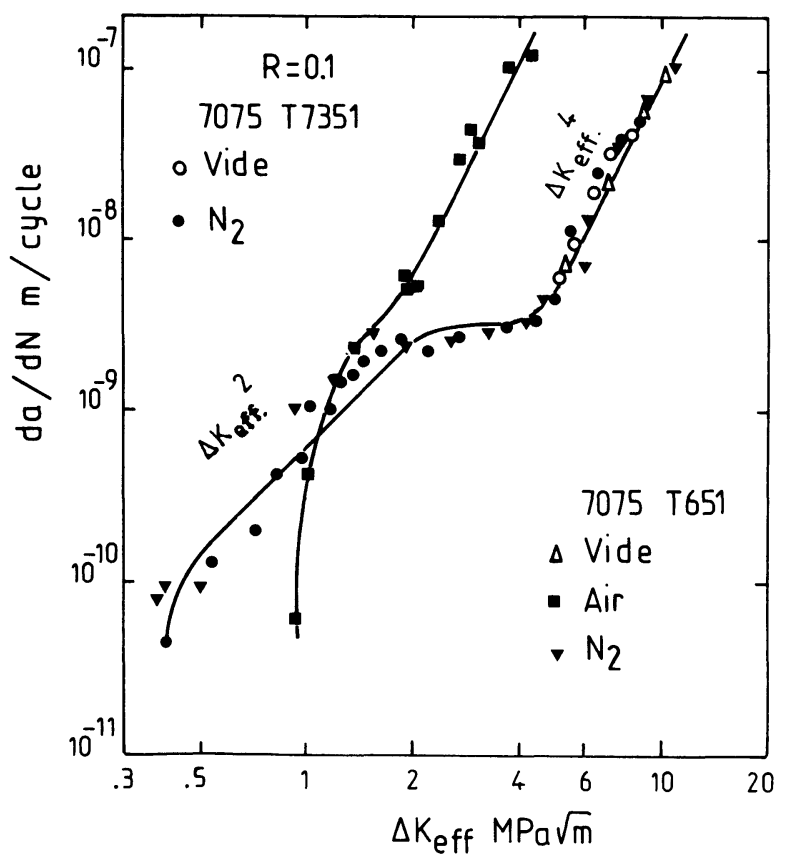

Fig. 7. $-\mathrm{d} a / \mathrm{d} N-\Delta K_{\text {eff }}$ à l'air, sous vide et dans l'azote (30 ppm $\mathrm{H}_{2} \mathrm{O}$ ).

[d $a / \mathrm{d} N$ vs. $\Delta K_{\text {eff }}$ in air, vacuum and nitrogen $+30 \mathrm{ppm}$ $\mathrm{H}_{2} \mathrm{O}$.]
- lorsque la vitesse $\mathrm{d} a / \mathrm{d} N$ est inférieure à $10^{-8} \mathrm{~m} /$ cycle, on observe l'apparition d'une propagation assistée par l'environnement qui peut être décrite à l'aide d'une loi de la forme :

$$
\frac{\mathrm{d} a}{\mathrm{~d} N}=B \frac{\Delta K_{\mathrm{eff}}^{2}}{\mu \sigma}
$$

correspondant au modèle théorique d'une propagation contrôlée par le $\triangle$ CTOD [34]. Un mécanisme de fissuration assistée par l'hydrogène a été suggéré $[7,31]$ sur la base des modèles proposés notamment par Achter [35], Bowles [36] et Wei et al. [37]. L'hydrogène produit par dissociation de la vapeur d'eau adsorbée en pointe de fissure peut diffuser dans la zone plastifiée par drainage par des dislocations au cours de la déformation cyclique [38]. L'apparition de ce mécanisme ne peut s'effectuer qu'à des vitesses suffisamment basses pour que le temps nécessaire à la diffusion soit requis mais également à des niveaux de contrainte suffisamment faibles pour que la déformation soit localisée dans un volume assez réduit (à l'échelle du grain) et qu'une concentration critique d'hydrogène soit atteinte. Cet effet de fragilisation est cohérent avec l'observation de valeurs de $\Delta K_{\text {eff }}$ seuil très faibles sous environnement actif. D'autres travaux ont montré ultérieurement que cette analyse pouvait s'appliquer aux aciers $[19,39]$ et au cuivre [40].

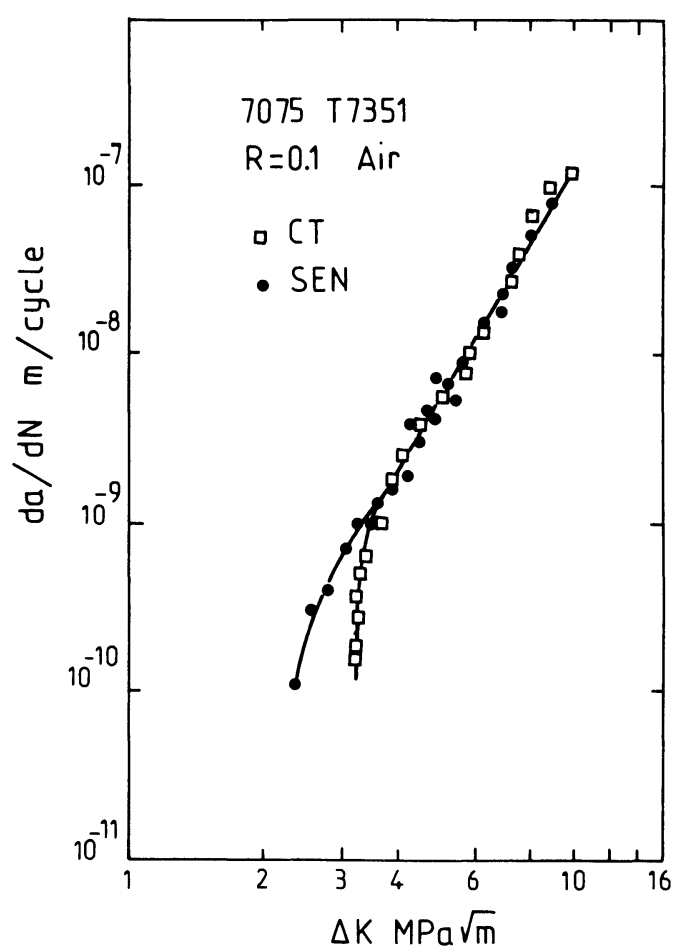

Fig. 8. $-\mathrm{d} a / \mathrm{d} N-\Delta K$ à l'air pour l'alliage 7075. Comparaison des résultats obtenus sur éprouvettes SEN et CT.

$[\mathrm{d} a / \mathrm{d} N$ vs. $\Delta K$ in air the $7075 \mathrm{~T} 7777351$. Comparison of results on SEN and CT specimens.] 


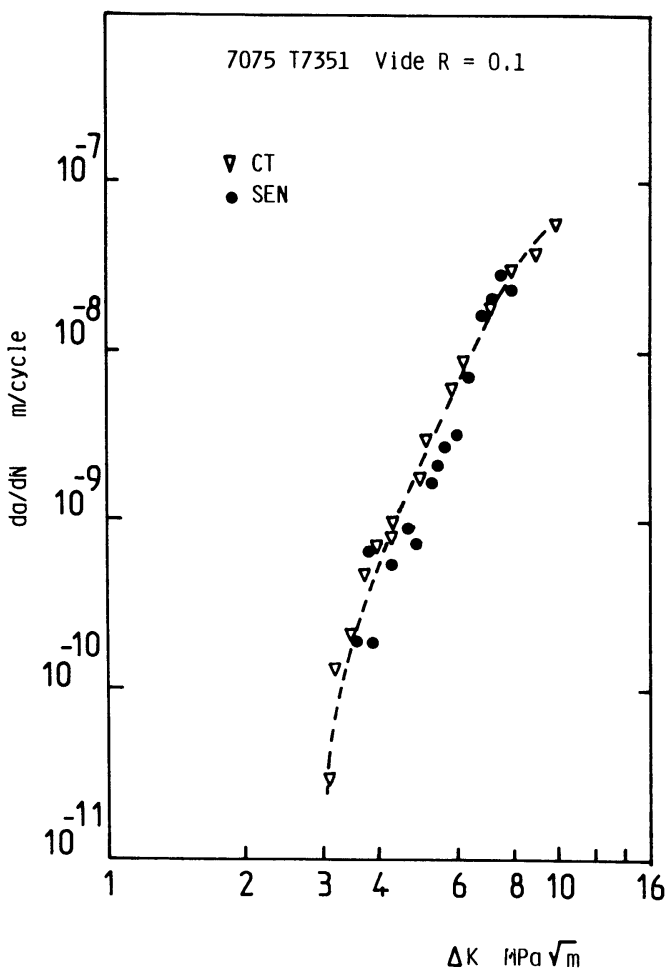

Fig. 9. $-\mathrm{d} a / \mathrm{d} N-\Delta K_{\text {eff }}$ sous vide pour l'alliage 7075 T7351. Comparaison des résultats sur éprouvettes SEN et CT.

[d $a / \mathrm{d} N$ vs. $\Delta K_{\text {eff }}$ in vacuum for the $7075 \mathrm{~T} 7351$ alloy. Comparison of results on SEN and CT specimens.]

On peut remarquer que, selon la théorie de Griffith [41] l'énergie $G$ nécessaire à l'avancée d'une fissure en mode 1 d'ouverture s'écrit en état de déformations planes (prédominant au seuil) :

$$
G=\frac{K_{1}^{2}}{E}\left(1-\nu^{2}\right)
$$

$E$ : module d'Young,

$\nu$ : coefficient de Poisson.

Dans les matériaux fragiles, une fissure pourra se propager dès lors que $G$ sera supérieure à $2 \gamma, \gamma$ étant l'énergie de surface libre. Le seuil théorique d'un matériau fragile peut alors être considéré comme suit :

$$
\Delta K_{\mathrm{S}}=\sqrt{\frac{2 \gamma E}{1-\nu^{2}}}
$$

En considérant la valeur de $\gamma$ pour l'aluminium fournie par la littérature, soit $0,98 \mathrm{~J} / \mathrm{m}^{2}$ [42], on obtient :

$$
\Delta K=0,4 \mathrm{MPa} \sqrt{m}
$$

Cette valeur est comparable à celle du seuil de $\Delta K_{\text {eff }}$ sous azote contenant des traces d'eau, c'est-àdire dans une atmosphère humide fragilisante. Elle paraît caractéristique du couple aluminium-vapeur d'eau.

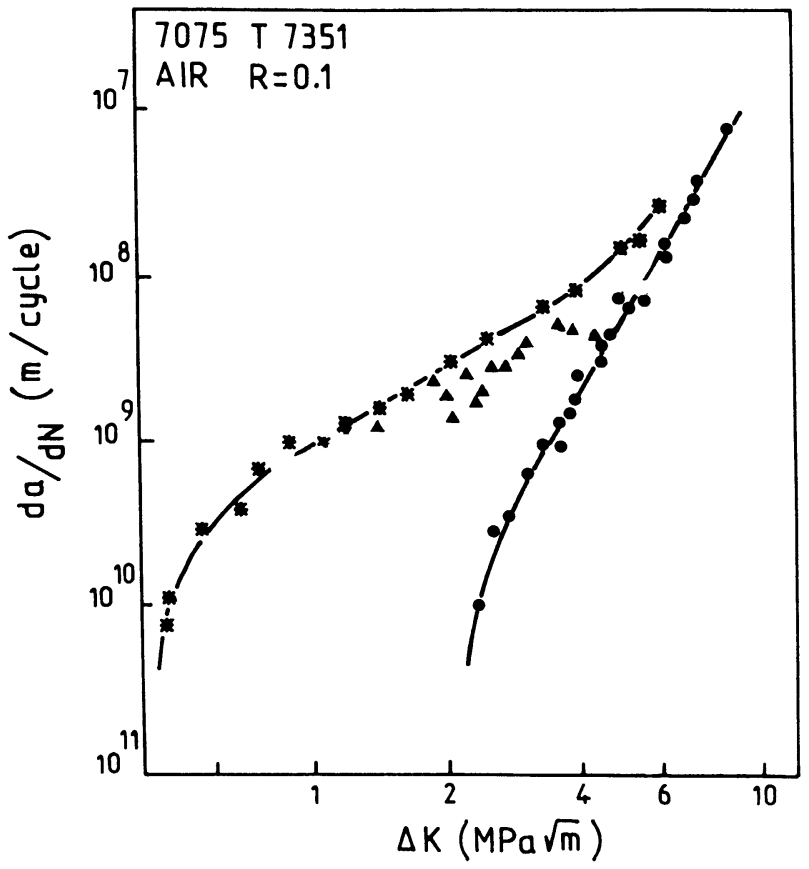

Fig. 10. - Alliage 7075 T7351, éprouvette SEN : Courbes $\mathrm{d} a / \mathrm{d} N-\Delta K(\bullet)$ et $\mathrm{d} a / \mathrm{d} N-\Delta K_{\text {eff }}(*)$ pour des essais à l'air à $R=0,1$.

[7075 T7351 alloy, SEN specimen. $\mathrm{d} a / \mathrm{d} N$ vs. $\Delta K$ and d $a / \mathrm{d} N$ vs. $\Delta K_{\text {eff }}$ relationships for tests in air at $R=0.1$.]

\section{Propagation des fissures courtes bidimension-} nelles.

4.1 INFLUENCE DE L'ENVIRONNEMENT. - Des essais de fissuration, ont été effectués à l'air et sous vide sur des éprouvettes de type CT de l'alliage 7075 T651. Après essai de seuil à $\Delta K$ décroissant à l'air qui correspond à une longueur de fissure de $24 \mathrm{~mm}$, le sillage plastique a été réduit progressivement par usinage par électroérosion comme l'indique la figure 2. Au cours de cette opération, le facteur d'intensité de contrainte d'ouverture $K_{\text {ouv }}$ a été mesuré en chargeant l'éprouvette au niveau seuil et sans progression de la fissure. La figure 11 représente les variations du facteur $K_{\text {ouv }}$ en fonction de la longueur de fissure restant après usinage. La valeur de $K_{\text {ouv }}$ diminue nettement lorsque la longueur du sillage devient inférieure à environ $2 \mathrm{~mm}$; quand celle-ci atteint $0,11 \mathrm{~mm}$, il n'y a plus de fermeture détectable par nos moyens de mesure. Ainsi après un essai de seuil, les contraintes de pression qui s'appliquent sur le sillage plastifié et provoquent la fermeture de la fissure, apparaissent essentiellement concentrées sur les $2 \mathrm{~mm}$ situés juste en amont de la pointe de la fissure. 


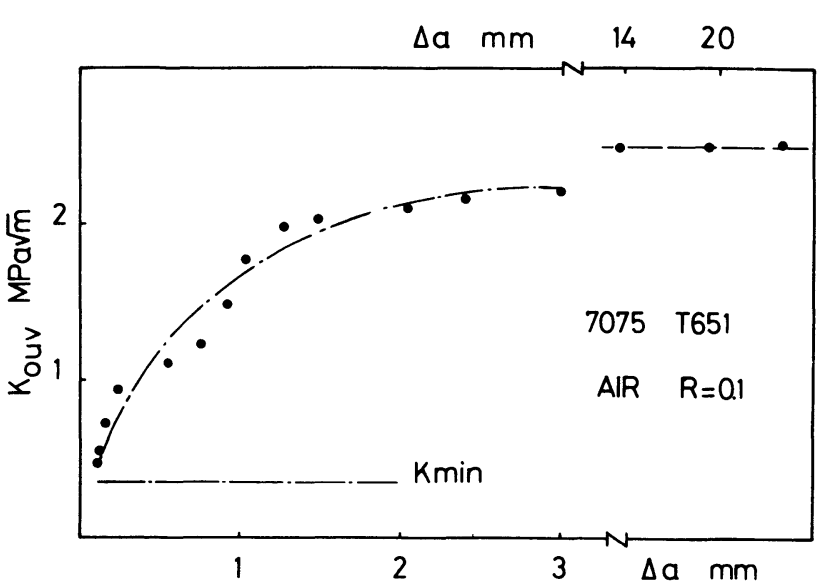

Fig. 11. - 7075 T651, éprouvette CT à l'air. Variations de $K_{\text {ouv }}$ en fonction de la longueur $\Delta a$ restant après usinage du sillage plastique.

[7075 T651, CT specimen in air. Variations of $K_{\text {ouv }}$ versus the residual crack length $\Delta a$ as obtained from machining of the long crack wake.]

La propagation de la fissure courte ainsi obtenue et dont la longueur initiale est de $0,11 \mathrm{~mm}$, est représentée sur la figure 12 comparativement à l'essai de seuil à l'air pour une fissure longue sur le même type d'éprouvette. Le début de la fissuration à une vitesse de $3 \times 10^{-9} \mathrm{~m} /$ cycle est observé pour une valeur de $\Delta K=2,5 \mathrm{MPa} \sqrt{m}$ nettement inférieure au seuil de la fissure longue soit $3,2 \mathrm{MPa} \sqrt{m}$. La recherche de la nouvelle valeur du seuil a été conduite en réduisant la charge par palier de $5 \%$. Une valeur de $2 \mathrm{MPa} \sqrt{m}$ a été obtenue pour une longueur fissurée de $0,82 \mathrm{~mm}$, la fissure n'ayant pas bougé après plus de $10^{6}$ cycles. La fissuration a été ensuite poursuivie après une légère augmentation de la charge. Pour une longueur $\Delta a \sim 2 \mathrm{~mm}$ un régime de propagation similaire à celui d'une fissure longue a été observé. Ces résultats sont en accord avec des observations faites par ailleurs [46, 48] selon la même technique expérimentale initialement proposée par Minakawa et al. [46].

La même éprouvette a été ensuite sollicitée sous vide. Après réusinage du sillage plastifié, une longueur résiduelle de $0,17 \mathrm{~mm}$ a été obtenue. La figure 12 compare le comportement sous vide et à l'air des fissures courtes et longues sur éprouvettes CT. La propagation initiale sous vide s'effectue à une vitesse de $3 \times 10^{-10} \mathrm{~m} /$ cycle pour une valeur de $\Delta K$ de $3,8 \mathrm{MPa} \sqrt{m}$ nettement inférieure au seuil des fissures longues sous vide soit $5,2 \mathrm{MPa} \sqrt{m}$. Comparativement à l'essai à l'air, la vitesse initiale sous vide s'avère 10 fois plus lente pour un $\Delta K$ initial supérieur de $50 \%$.

Après cette phase de démarrage, la charge a été abaissée de $5 \%$ et aucune évolution de la propagation n'a été observée après plus de $10^{6}$ cycles. Après réaugmentation de la charge, la propagation ulté-

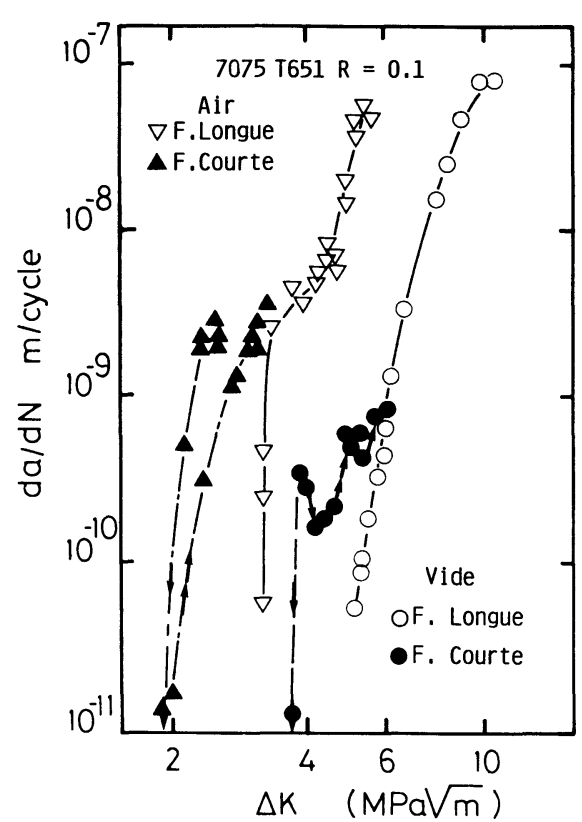

Fig. 12. - 7075 T651, éprouvette CT. Vitesses de propagation $\mathrm{d} a / \mathrm{d} N$ en fonction de $\Delta K$ sous vide et à l'air pour des fissures courtes comparées au comportement des fissures longues.

[7075 T651, CT specimen. $\mathrm{d} a / \mathrm{d} N$ versus $\Delta K$ relationships in vacuum and in air for small cracks compared to long crack data.]

rieure s'effectue dans un premier temps à vitesse décroissante jusqu'à un minimum (Fig. 12) atteint après un accroissement de longueur de $0,1 \mathrm{~mm}$. Puis la propagation se poursuit à vitesse croissante avec toutefois des fluctuations assez importantes. On peut noter une relation entre ces fluctuations et la taille du grain dans le sens de la propagation (50 à $60 \mu \mathrm{m})$. Ces observations sont en accord avec celles faites par ailleurs [47] qui montrent notamment que la décroissance initiale de la vitesse intervient au niveau du premier joint de grain. Sous vide le régime correspondant au comportement fissures longues est atteint pour $\Delta a \sim 1 \mathrm{~mm}$, valeur moitié plus faible qu'à l'air.

Sur la figure 13 le passage de l'air au vide est indiqué par les points de microdureté. On peut voir que sous vide, la propagation de type cristallographique associée au glissement monoplanaire, se caractérise par des changements de direction de propagation au niveau des joints de grains qui font ainsi localement obstacle à la progression de la fissure. Les faciès de rupture observés au microscope électronique à balayage (Figs. 14a, b et c) confirment le net changement de mécanisme de propagation lors du passage de l'air au vide. Le relief des surfaces rompues est très accidenté sous vide et l'on constate l'existence de facettes correspondant à la taille des grains. 


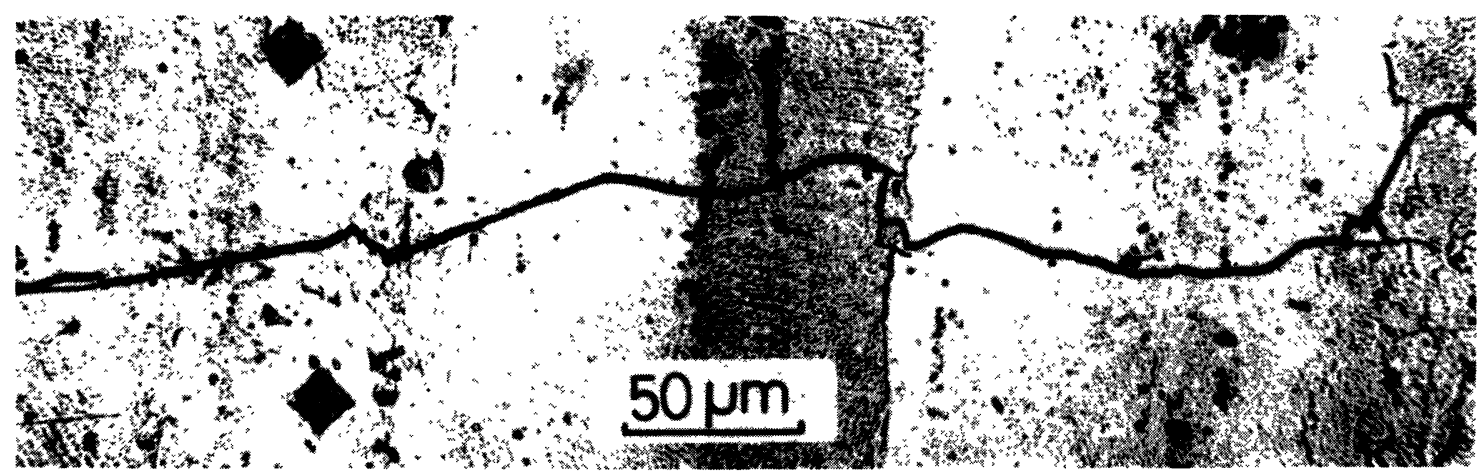

Fig. 13. - 7075 T651. Variations du profil de propagation de la fissure lors du passage air-vide répéré par les points de microdureté.

[7075 T651. Change in the crack path observed at the air-vacuum transition marked by two microhardeness indents.]

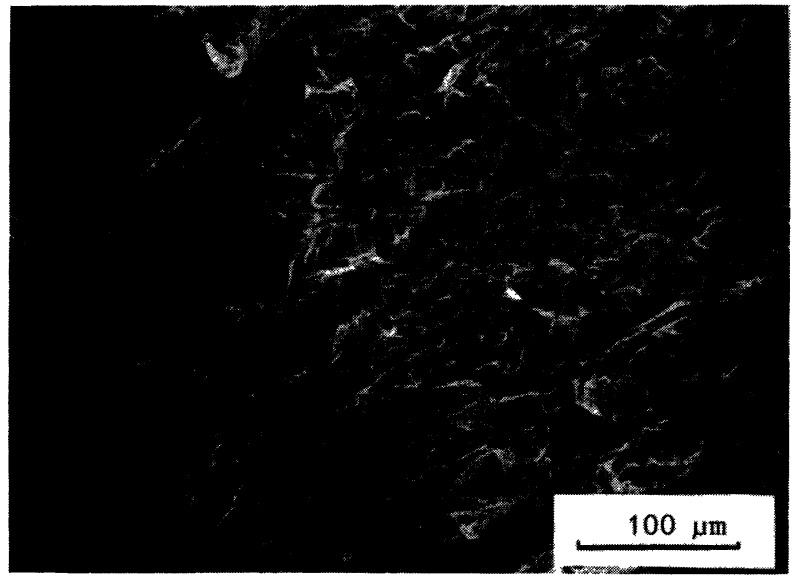

a)

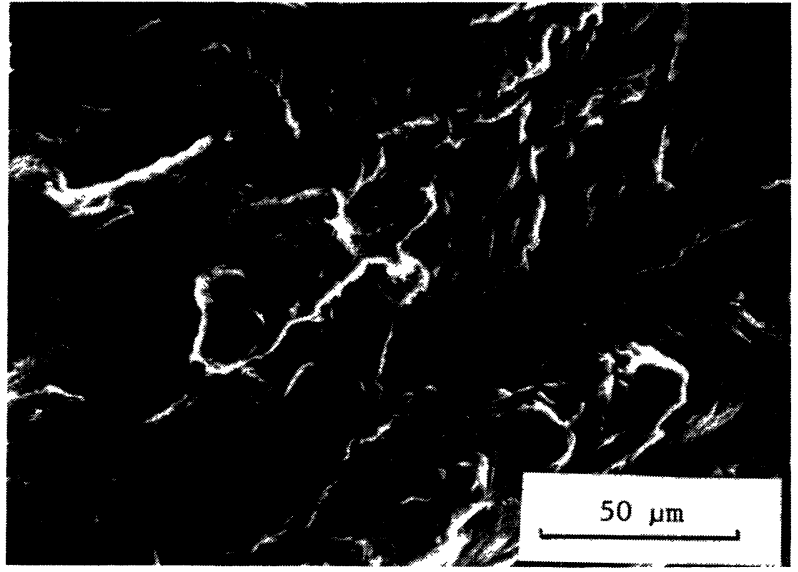

b)

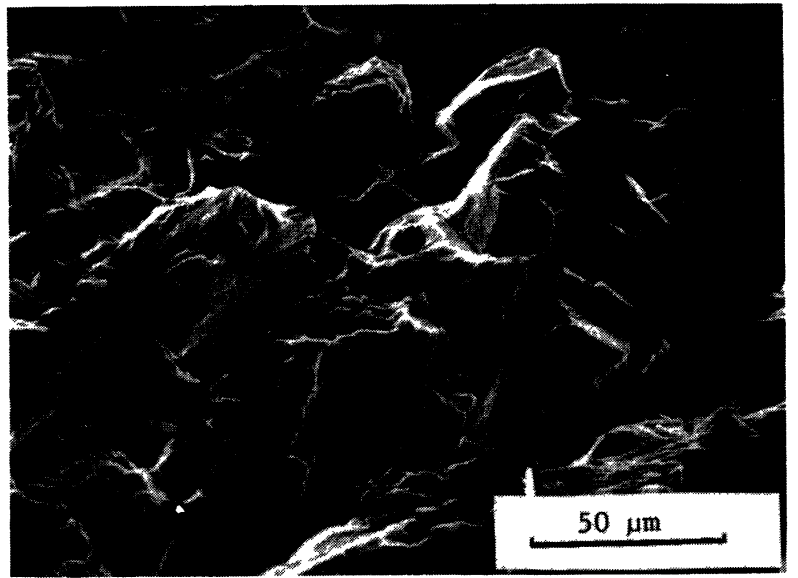

c)

Fig. 14. - Surface de rupture d'une fissure courte bidimensionnelle dans l'alliage 7075 T651 : a) passage air-vide ; b) propagation à l'air ; c) propagation sous vide.

[Microfractographic of the fracture surface of a short fatigue crack grown in the 7075 T651 alloy: a) air-vacuum transition ; b) propagation in air ; c) propagation in vacuum.] 
La figure 15 représente l'évolution du facteur $K_{\text {ouv }}$ en fonction de la longueur de fissure pour les deux environnements. Ces résultats montrent clairement que la fermeture augmente avec la longueur fissurée. Dans le cas de l'essai à l'air, le facteur $K_{\text {ouv }}$, dont la variation est comparable à celle observée par usinage du sillage plastifiée (Fig. 11), atteint une valeur maximum (stabilisation de l'effet de fermeture) pour une longueur de fissure $\Delta a \sim 2 \mathrm{~mm}$. Sous vide l'augmentation de $K_{\text {ouv }}$ est plus rapide et conduit à une valeur maximum stabilisée pour une longueur de fissure de l'ordre de $1 \mathrm{~mm}$. Ce développement plus rapide de l'effet de fermeture sous vide peut être relié à la rugosité des surfaces rompues.

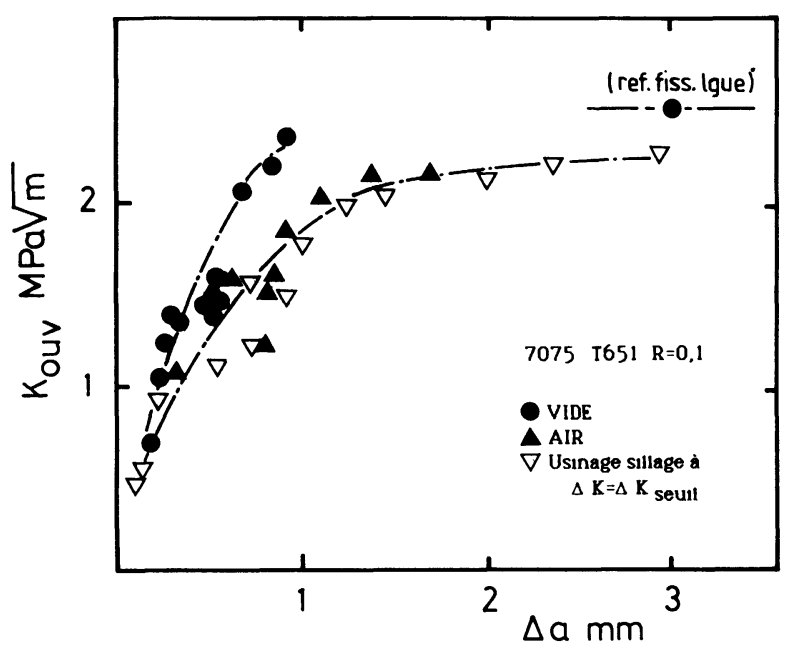

Fig. 15. - 7075 T651. Variations de $K_{\text {ouv }}$ en fonction de la longueur de fissure $\Delta a$ à l'air et sous vide.

[7075 T651. $K_{\text {ouv }}$ versus $\Delta a$ for short cracks in air and in vacuum.]

La figure 16 présente les courbes $\mathrm{d} a / \mathrm{d} N-\Delta K_{\text {eff }}$ pour les fissures courtes et longues, à l'air et sous vide.

Les résultats à l'air montrent que l'effet fissure courte est totalement pris en compte lorsque l'on utilise $\Delta K_{\text {eff }}$ pour décrire la propagation. Ce résultat est en accord avec les observations faites par ailleurs dans différents matériaux [10-11].

Les résultats sous vide conduisent à la même conclusion, $\Delta K_{\text {eff }}$ rationnalisant également le comportement des fissures courtes et longues sous environnement inerte. Il reste que la figure 16 souligne l'existence d'une influence de l'environnement très importante sur la propagation des fissures courtes. Cette influence, identique à celle observée pour les fissures longues près du seuil en termes de $\Delta K_{\text {eff }}$, s'avère en pratique encore plus marquée pour les fissures courtes lorsque la fermeture joue un faible rôle.

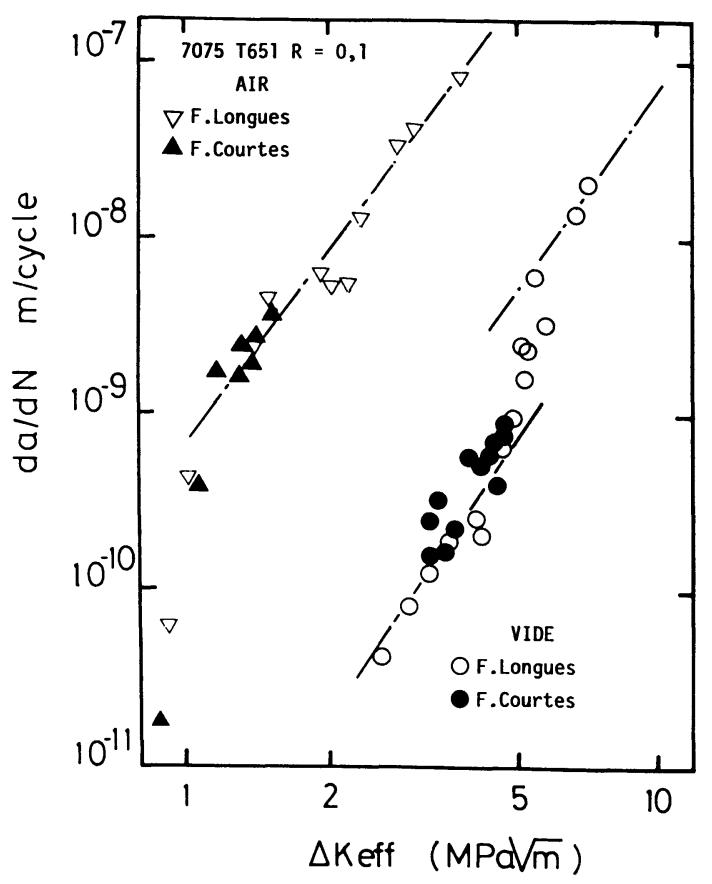

Fig. 16. - $7075 \mathrm{~T} 651$, éprouvette CT. $\mathrm{d} a / \mathrm{d} N$ en fonction de $\Delta K_{\text {cff }}$ pour les fissures courtes et longues à l'air et sous vide.

[7075 T651, CT specimen. $\mathrm{d} a / \mathrm{d} N$ vs. $\Delta K_{\text {eff }}$ relationship for small and long cracks in air and in vacuum.]

Enfin la figure 16 montre que les modélisations de la propagation développées pour les fissures longues près du seuil (chap. précédent) peuvent être étendues au cas des fissures courtes avec, à l'air ambiant, un mécanisme de fissuration assisté par l'hydrogène provenant de la vapeur d'eau adsorbée et sous vide un mécanisme d'endommagement par écrouissage cyclique.

4.2 Influence de la microstructure. - Des essais ont été effectués à l'air ambiant sur éprouvettes type SEN de l'alliage 7075 T7351. Après fissuration jusqu'au seuil (atteint pour une longueur de l'ordre de $4 \mathrm{~mm}$ ), l'éprouvette a ensuite été usinée comme précédemment (Fig. 1) pour obtenir une fissure traversante ayant une longueur initiale de $0,4 \mathrm{~mm}$.

La figure 17 compare la propagation de la fissure courte à celle de la fissure longue issue de la figure 8 . On observe que la fissure courte se propage classiquement plus vite que la fissure longue. La valeur initiale de $\Delta K \sim 1,4 \mathrm{MPa} \sqrt{m}$ est inférieure au seuil des fissures longues (2,4 MPa $\sqrt{m}$ (Fig. 8)). La propagation initiale est proche de la courbe $\mathrm{d} a / \mathrm{d} N-\Delta K_{\text {eff }}$ issue de la figure 10 , ce qui est en accord avec une absence de fermeture. Un comportement analogue à celui de la fissure longue est obtenu pour une longueur voisine de $2 \mathrm{~mm}$. Ces résultats sont en accord avec ceux obtenus sur l'état T651. 


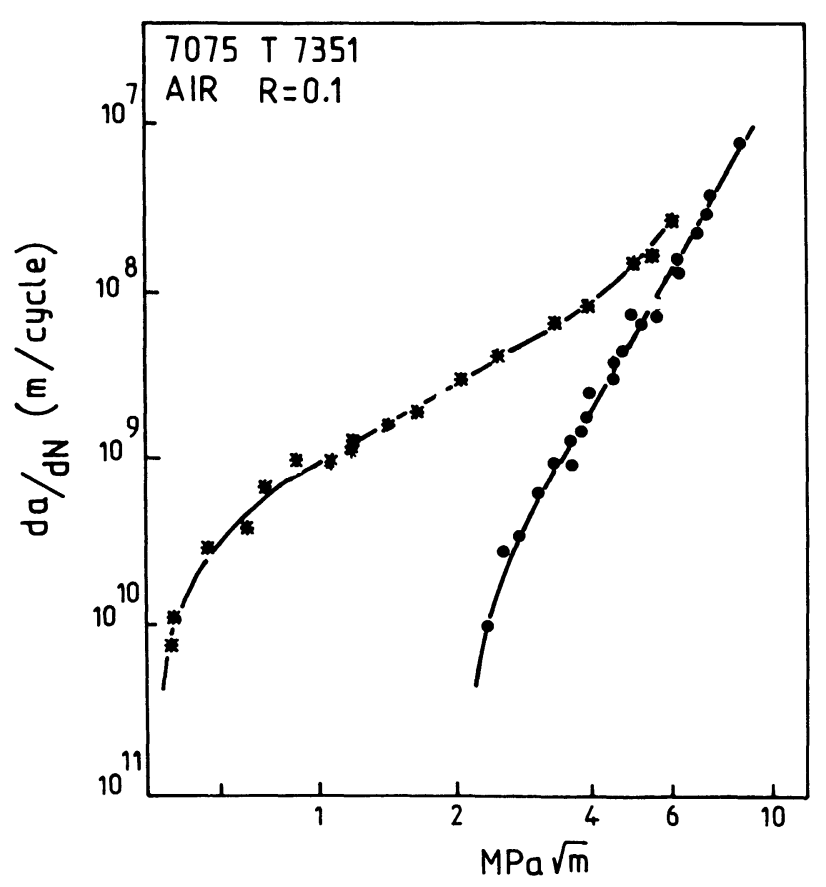

Fig. 17. - Propagation d'une fissure courte à l'air dans l'alliage 7075 T7351 (A) comparée à celle d'une fissure longue (Fig. 10). Eprouvette SEN.

[Fatigue propagation of a short crack in air $(\Delta)$ in a SEN specimen of the $7075 \mathrm{~T} 7351$ alloy compared to propagation of a long crack (from Fig. 10).]

Dans le domaine de vitesse où la propagation des fissures a été observée, les essais de références sur fissures longues ont montré l'absence de différence significative entre éprouvette SEN et CT pour l'état T7351. Une comparaison de la propagation des fissures courtes peut donc être faite à l'air pour les deux nuances.

La figure 18 montre une faible influence de la microstructure sur la propagation à l'air des fissures courtes dans l'intervalle de vitesse correspondant. Les mesures correspondant à l'essai de seuil sur l'alliage 7075 T651 ont été éliminées afin de clarifier la comparaison. Il reste que des essais complémentaires, notamment sous vide, sont nécessaires pour préciser le rôle intrinsèque de la microstructure sur «l'effet fissure courte».

\section{Conclusions.}

La propagation en fatigue de fissures bidimensionnelles artificielles de petite longueur a été comparée à la propagation des fissures longues dans un alliage 7075 élaboré dans deux états de revenu, T651 et T7351.

«L'effet fissure courte», correspondant à une propagation plus rapide que celle d'une fissure

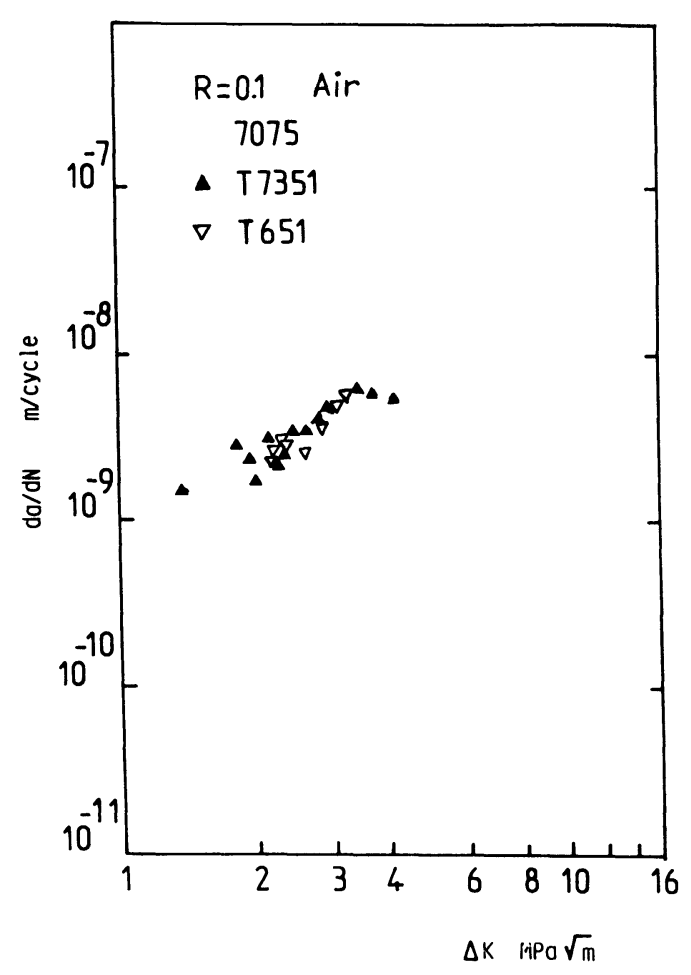

Fig. 18. - Influence de la microstructure sur la propagation des fissures courtes et longues à l'air ambiant à $R=0,1$.

[Influence of microstructure of the propagation of short and long through cracks in ambient air at $R=0.1$.]

longue dans les mêmes conditions de sollicitation, est attribué dans le cas étudié à une variation de l'amplitude de l'effet de fermeture avec la longueur de la fissure. Les mesures effectuées ont montré que la longueur de fissure où l'on observe cet effet dépend peu de la microstructure mais s'avère très sensible à l'environnement.

Pour chaque environnement la propagation des fissures bidimensionnelles longues et courtes peut être décrite à l'aide d'une loi unique reliant la vitesse $\mathrm{d} a / \mathrm{d} N$ au facteur d'intensité de contrainte effectif $\Delta K_{\text {eff }}$ (c'est-à-dire après soustraction des effets mécaniques de fermeture).

Les mécanismes contrôlant la propagation des fissures longues près du seuil, adsorption de vapeur d'eau et fragilisation par l'hydrogène en atmosphère humide d'une part, endommagement par écrouissage cyclique sous vide d'autre part, gouvernent de façon identique la propagation des fissures courtes.

En l'absence de fermeture, les premiers stades de propagation des fissures courtes sont donc très sensibles à l'environnement, au même titre que les fissures longues pour des rapports de charge $R$ élevés. 
Bibliographie

[1] Gangloff R. P., Embrittlement by the localised crack environment, R. P. Gangloff Ed. TMS AIME (Warrendale, PA) 1984, p. 265.

[2] Gangloff R. P. (1985), Met. Trans. 16A (1985) 953.

[3] Gerdes C., Gysler A. and Lutjering G., Fatigue Crack Growth Thresholds Concepts, D. L. Davidson and S. Suresh Eds. TMS AIME (Warrendale, PA) 1984, p. 465.

[4] LANKFord J., Fatigue Engng. Mat. Struct. 6 (1983) p. 15.

[5] Zeghloul A. and Petit J., Fatigue Fract. Engng. Mat. Struct. 8 (1985) p. 341.

[6] Kirby R. B. and BeEvers C. J., Fatigue Engng. Mat. Struct. 1 (1979) p. 203.

[7] Petit J., Fatigue Crack Growth Thresholds Concepts, D. L. Davidson and S. Suresh Eds. TMS AIME (Warrendale, PA) 1984, p. 3. ·

[8] Vasudevan A. K. et Bretz P. E., Fatigue Crack Growth Thresholds Concepts, D. L. Davidson and S. Suresh Eds. TMS AIME (Warrendale, PA) 1984, p. 25.

[9] Mc Carver J. F. and Ritchie R. O., Mater. Sci. Engng. 55 (1982) 63.

[10] TANAKA K. and NakaI Y., Fatigue Engng. Mat. Struct. 6 (1983) 315.

[11] Breat J. L., Mudry F. and Pineau A., Fatigue Engng. Mat. Struct. 6 (1983) 349.

[12] James M. R. and Morris W. L., Met. Trans. 14A (1983) 153.

[13] Byrne J., Fatigue 87, R. O. Ritchie and E. A. Starke Jr. Eds. (EMAS Ltd, Warley, U.K.) 1987, p. 195.

[14] SONIAK F. and REMY L., Idem (1987) p. 351.

[15] BRown W. F. Jr and SRAWLEY J. E., ASTM STP 410 (1966).

[16] Нiвbiтt H. D., Int. J. Num. Meth. Engng. 11 (1977) 180.

[17] RicE J. R., ASTM STP 415 (1967) p. 247.

[18] Renaud P., Influence de la microstructure sur le comportement en fatigue d'un alliage d'aluminium 7075 , thèse de docteur de $3^{\mathrm{e}}$ cycle, Poitiers (1982).

[19] Kwon J. H., Influence de l'environnement sur le comportement en fatigue d'un acier E460 et d'un alliage léger 7075 près du seuil de fissuration, thèse de docteur d'université, Poitiers (1985).

[20] LAfarie-Frenot M. C., Etude de la fermeture des fissures de fatigue dans le vide: Influence des mécanismes de rupture, thèse de docteur ès sciences physiques, Poitiers (1986).

[21] Zeghloul A., Comparaison de la propagation en fatigue des fissures courtes et des fissures longues, thèse de docteur ès sciences physiques, Poitiers (1988).

[22] Petit J., Renaud P. and Violan P., Proceedings ECF4, K. L. Maurer and F. E. Matzer Eds. (EMAS Ltd, Warley, U.K.) 1982, p. 426.

[23] Renaud P., Violan P., Petit J. and Ferton D., Scripta Met. 16 (1982) p. 1311.

[24] SELINES R. J., The fatigue behavior of high strength aluminium alloy, Doc. of Sci. Massachusetts Institute of technology, U.S.A. (1975).

[25] WeErtman J., Int. J. Fracture 9 (1973) 125.

[26] SuRESH S., Crack deflection and fracture surface contact in fatigue: micromechanical models, Brown University report $n^{\circ}$ MRL.E. 153 (1984).

[27] Suresh S., Zamiski G. F. and Ritchie R. O., Met. Trans. 12A (1981) 1435.

[28] Vasudevan A. K. and Suresh S. (1982), Met. Trans. 13A (1982) 2271.

[29] TraceY D. (1976), Eng. Mat. and Techn. ASME 98 (1976) 146

[30] Suyitno B., thèse de Docteur d'Université de Poitiers, 1988 (à paraître).

[31] Zeghloul A., Etude de l'effet de l'environnement gazeux sur la fissuration par fatigue dans les alliages d'aluminium à haute résistance, thèse de Docteur Ingénieur, Poitiers (1980).

[32] Petit J. and Zeghloul A., Fatigue Thresholds, J. Bäcklund, A. F. Blom and C. J. Beevers (EMAS Ltd, Warley U.K.) 1982, p. 563.

[33] Westwood A. R. C. and Ahearn J. S., Physical Chemistry of the solid state: applications to metals and compounds, J. Lacombe Ed. (Elsevier Amsterdam) 32 (1984) 65.

[34] McClintock F. A., ASTM STP 415 (1967) p. 169.

[35] ACHTER M. R., Scripta Met. 2 (1968) 525.

[36] BOWLES C. Q., The role of environment frequency and wave shape during fatigue crack growth in aluminium alloys, Report L.R. 270, Delft University of technology, The Netherlands (1979).

[37] Wei R. P., PAO P. S., HART R. G., Weir T. W. and Simmons G. W., Met. Trans. 11A (1980) 151.

[38] Albrecht J., Bernstein I. M. and Thompson A. W., Met. Trans. 13A (1982) 811.

[39] Kwon J. H., Bignonnet A., Bouchet B. and Petit J., Fatigue 84, C. J. Beevers Ed. (EMAS Ltd, Warley U.K.) III (1984) 435.

[40] Marchand N., Ballon J. P. and Dickson J. I., « Near Threshold fatigue Crack Growth in Copper and Alpha-Brass: Grain size and environmental effects », 1988 (à paraître).

[41] Griffith A. A., Phil. Trans. Royal Soc. London, série A (1920) p. 163.

[42] Mura T. and Weertman J., Fatigue Crack Growth Thresholds Concepts, D. Davidson and S. Suresh Eds. TMS AIME (Warrendale PA) 1984, p. 531.

[43] Lankford J. and Davidson D. L., Acta Met. 8 (1983) 1273.

[44] Ranganathan N., Bouchet B. and Petit J. ASTM STP 948 (1984) p. 424.

[45] Davidson D. L., Fatigue Engng. Mat. Struct. 3 (1981) 229.

[46] Minakawa K., Nakamura M. et Mc Evily A. S., Scripta Metall. 18 (1984) 1371.

[47] Clement P., Angeli J. P. et Pineau A., Fat. Engng. Mater. Struct. 7 (1984) 251.

[48] Lefrancois A., Clement P. et Pineau A., Fat. Engng. Mater. Struct., proceedings of the Institution of Mechanical Engineers (15-16 Sept. 86, Sheffield, (U.K.), paper C 2346/86 (1984) p. 56. 